\title{
A 1.2V, 18mW, 10Gb/s SiGe Transimpedance Amplifier
}

\author{
Chihun Lee, Chia-Hsin Wu, and Shen-Iuan Liu \\ Graduate Institute of Electronics Engineering \& Department of Electrical Engineering \\ National Taiwan University, Taipei, Taiwan 10617, R. O. C.
}

\begin{abstract}
To the authors' knowledge, the first $1.2 \mathrm{~V}, 18 \mathrm{~mW}$, $10 \mathrm{~Gb} / \mathrm{s} \mathrm{SiGe} \mathrm{transimpedance} \mathrm{amplifier} \mathrm{(TIA)} \mathrm{is} \mathrm{presented}$ here. It has been realized in a $0.35 \mu \mathrm{m}$ SiGe process and its area is $0.45 \mathrm{~mm}^{2}$ with pads. Employing inductive series and shunt peaking techniques, the proposed TIA can achieve a transimpedance gain of $61.6 \mathrm{~dB} \Omega$ and the bandwidth of $7.4 \mathrm{GHz}$, while dissipating only $18 \mathrm{~mW}$ with $1.2 \mathrm{~V}$ supply. With an equivalent photodiode capacitance of $0.15 \mathrm{pF}$, this TIA shows the input referred noise current density of $22 \mathrm{pA} / \sqrt{\mathrm{Hz}}$.
\end{abstract}

\section{Introduction}

The growing popularity of multimedia applications demands high data rate wired telecommunication systems. Many existing wired systems require $10 \mathrm{~Gb} / \mathrm{s}$ analog front-ends, such as $\mathrm{OC} 192$ for telecommunications and $10 \mathrm{~Gb} / \mathrm{s}$ Ethernet for data communications. The key requirements for designing a high-speed front-end are: 1) to integrate both the high-speed analog front-end and digital back-end and 2) low voltage and low power. Traditionally, analog RF front-end utilized high $f_{T}$ transistors of GaAs. However, GaAs RF circuits could not be integrated with succeeding CMOS digital processors. With the past decade, there is a significant effort to implement the multi-gigabits optical front-ends [1]-[5] in BiCMOS and CMOS technologies. It can provide the advantages of high integration and low cost.

Conventional bandwidth-enhancement techniques, such as the regulated cascode topology [2] [3] and the negative feedback configuration [1], will suffer the limitation of the supply voltage. When the deep submicron technology advances further, the low supply voltage is indispensable. In this paper, a low-voltage and low power transimpedance amplifier (TIA) utilizes the inductive series and shunt peaking techniques to improve the overall bandwidtb. To save the power dissipations, this proposed TIA can provide a high transimpedance gain without a power-hungry buffer because it uses the same gain stage to match a 50 -ohm resistive load directly. A $1.2 \mathrm{~V}, 18 \mathrm{~mW}$, $10 \mathrm{~Gb} / \mathrm{s}$ SiGe transimpedance amplifier is implemented in a $0.35 \mu \mathrm{m} \mathrm{SiGe} 3 \mathrm{p} 3 \mathrm{~m}$ process. Compared to the current high-speed TIAs [1]-[6], the proposed one can achieve the lowest supply voltage and lowest power consumption.

\section{Circuit design}

\section{A. Low-voltage amplifier}

To develop low-voltage analog and digital circuits is

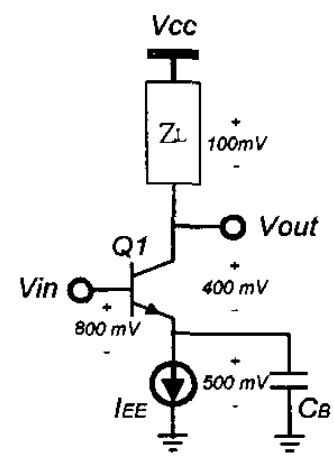

(a)

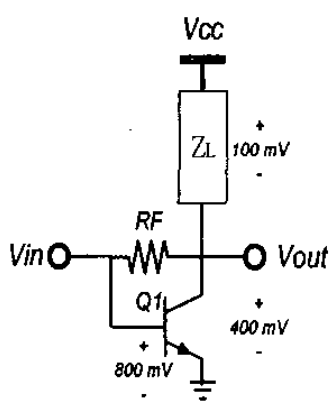

(b)
Fig. 1 Common emitter amplifier (a) conventional topology (b) proposed low-voltage one.

of great interest. In [7], it is described that bipolar circuits have the innate DC headroom problem. For the bipolar transistors with a constant current density, the base-emitter voltage $\left(\mathrm{V}_{\mathrm{BE}}\right)$ does not decrease with the scale-down technology and it has a turn-on voltage of $800 \mathrm{mV}$. Besides, a minimum collector-emitter voltage $\left(\mathrm{V}_{\mathrm{CE}}\right)$ around $400 \mathrm{mV}$ can protect high-speed bipolar transistors against heavy saturation. Under a supply voltage of $1.2 \mathrm{~V}$, the classical cascode amplifier using bipolar devices is forbidden. Fig. 1 illustrates two common emitter amplifiers. The evaluation of their minimum supply voltage for a low supply voltage of $1.2 \mathrm{~V}$ and high-speed operation are considered. In the conventional topology of Fig. 1(a), it uses a bypass capacitor $C_{B}$ and a tail current source $I_{E E}$ that may occupy $500 \mathrm{mV}$ headroom in experience. Obviously, the sum of $\mathrm{V}_{\mathrm{BE}}$ in $\mathrm{Q} 1$ and the voltage across the tail current source will exceed the supply voltage. Fig. 1(b) represents a shunt-shunt feedback amplifier with $R_{F}$, which can have a dc self bias and provide input matching. When the voltage swings across $\mathrm{Z}_{\mathrm{L}}$ maintain as small as $100 \mathrm{mV}$, it is sufficient to make the amplifier operated in the supply voltage below $1 \mathrm{~V}$ operation.

Furthermore, the voltage gain for two common emitter amplifiers in Figs. 1(a) and (b) can be calculated. For the conventional common emitter amplifier in Fig. 1(a) with the bypass capacitor, $\mathrm{C}_{\mathrm{B}}$, the small-signal voltage gain can be

$$
A=\frac{v_{o u t}}{v_{\text {in }}}=-g_{m} Z_{L} \text {, }
$$

where $g_{m}$ is the transconductance. Since $g_{m}=I_{C} / V_{T}$, eq. (1) can be sequentially simplified as follows: 


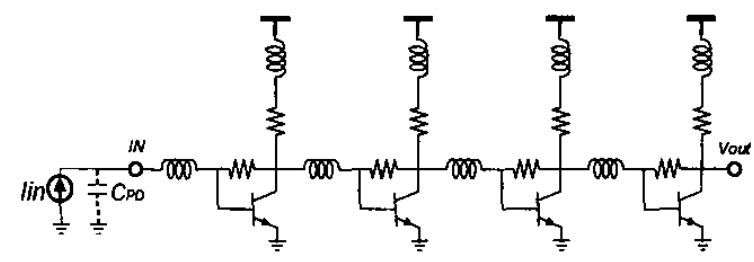

Fig. 2 The proposed TIA

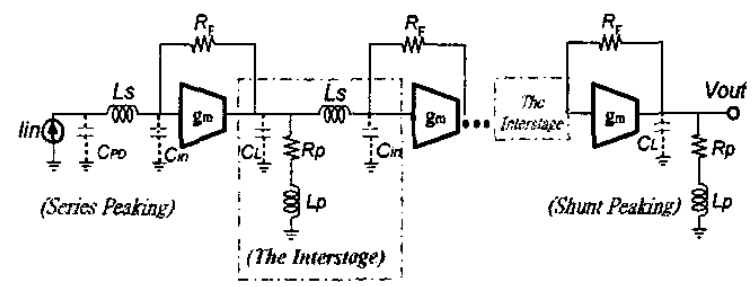

Fig. 3 The small-signal model for the proposed TIA

$$
\left|\frac{v_{o u t}}{v_{\text {in }}}\right|=\frac{I c \cdot Z_{L}}{V_{T}}
$$

where $\mathrm{V}_{\mathrm{T}}=k T / q$. In eq. (2), the sufficient voltage across $Z_{L}$ can increase the gain and it is especially important for low-voltage amplifiers. Supposed that the impedance of $Z_{L}$ is much smaller than $R_{F}$, the low-voltage topology in Fig. 1(b) can reach a similar voltage gain as Fig. 1(a). By means of Miller's theorem, the input impedance of the amplifier in Fig. 1(b) can be

$$
R_{i n}=\frac{R_{F}}{1-A}=\frac{R_{F}}{1+g_{m} Z_{L}}
$$

Furthermore, this input impedance can be designed to match the input $50 \mathrm{ohm}$ termination according to eq. (3).

\section{B. The proposed TIA}

Based on Fig. 1(b), the proposed TIA is presented in Fig. 2 by using the inductive series and shunting peaking techniques [8]. Compared to the conventional TIAs [1-5], the proposed one can have several advantages: self-bias operation, low input impedance, and high gain for low-voltage and low-power applications.

The small-signal model for the proposed TIA is shown in Fig. 3. An inductor $\mathrm{L}_{\mathrm{S}}$ can be inserted between a photodiode $\mathrm{C}_{\mathrm{PD}}$ and a shunt-shunt feedback amplifier in order to increase the bandwidth. In practical, the core amplifier exhibits one pole system. Thus, series peaking with a damping factor of $1 / \sqrt{2}$ can multiply the bandwidth by 1.4 times. The inductive shunting peaking technique is used in high-speed output buffers to ease the trade-off between output matching, speed, and voltage headroom. An inductor $L_{p}$ in series with the resistance $R_{P}$ can enlarge the

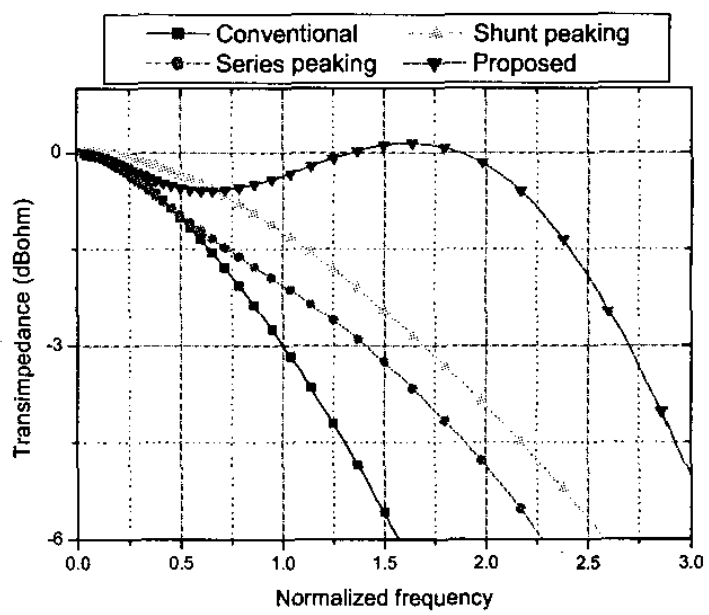

Fig. 4 Simulated bandwidths for the conventional TIA, the series peaking one, the shunting peaking one and the proposed one, respectively.

bandwidth. Therefore, the shunt peaking technique can also have a flat response to enhance the bandwidth by a factor of 1.7. The simulated bandwidth enhancement factors for the conventional TIA, the series peaking one, the shunting peaking one and the proposed one, respectively, are shown in Fig. 4. Compared to the conventional amplifier, the bandwidth enhancement factor for the conventional TIA, the series peaking one, the shunting peaking one and the proposed one is $1.4,1.7$ and 2.7 , respectively. Obviously, the proposed TIA can have a larger bandwidth enhancement factor.

\section{Devices selection for the proposed TIA}

In considerations of $\mathrm{DC}$ biasing, the selection for the bipolar transistors should trade off among headroom of $\mathrm{V}_{\mathrm{CE}}$, collector current density $(\mathrm{Jc})$, unity-gain frequency $\left(f_{t}\right)$, gain, noise, and power consumptions. In addition, a bipolar device with $\mathrm{Jc}$ of $640 \mathrm{uA} / \mathrm{um}^{2}$ is selected for the high-speed date rates of $10 \mathrm{~Gb} / \mathrm{s}$. Each identical bipolar transistor in the proposed TIA obtains $1.1 \mathrm{v}$ headroom and achieves the current gain of 160 with $f_{t}$ of $40 \mathrm{GHz}$.

\section{Experimental results}

The proposed TIA has been implemented in a 0.35 um $3 \mathrm{P} 3 \mathrm{M}$ SiGe process and measured by on-wafer probing. Fig. 5 shows two TIAs: the top one is the proposed TIA with eight inductors and the bottom one is a series peaking TIA with four inductors. Due to high-speed considerations, the small inductors below $1 \mathrm{nH}$ are used. Two core circuits separately occupy the area of $900 \mathrm{um} \times 400 \mathrm{um}$ and $900 \mathrm{um} \times 200 \mathrm{um}$, respectively. Operating from the supply voltage of $1.2 \mathrm{~V}$, both of them consume the same current of $15 \mathrm{~mA}$. 


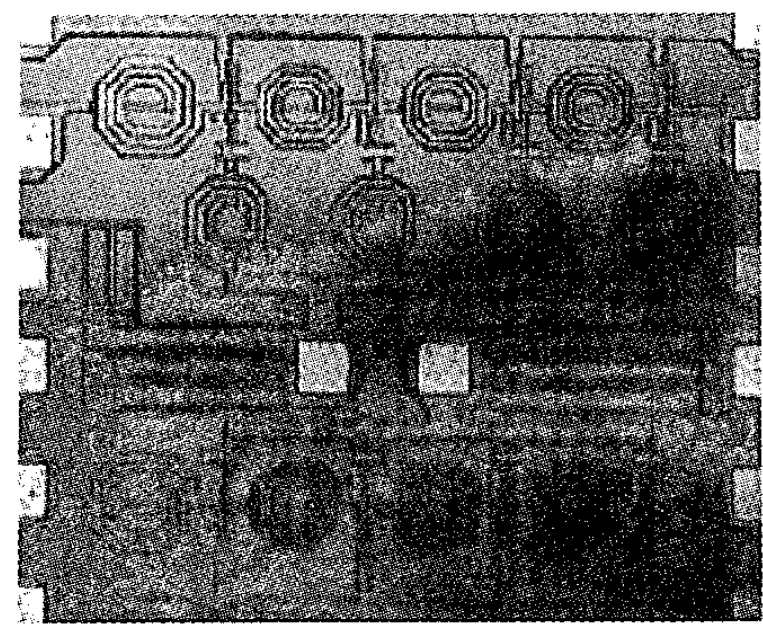

Fig. 5 The die micrograph for the proposed TIA (top) and the series peaking one (bottom).

With an equivalent photodiode capacitance of $0.15 \mathrm{pF}$, both TIAs are measured by on-wafer probing. For a supply voltage of $1.2 \mathrm{~V}$, the measured transimpedance gain for the proposed TIA and the series peaking one is 61.6dB $\Omega$ and $58 \mathrm{~dB} \Omega$, respectively, as shown in Fig. 6(a). The measured bandwidth, $\mathrm{f}_{-3 \mathrm{~dB}}$, for the proposed TIA and the series peaking one is $7.4 \mathrm{GHz}$ and $4.4 \mathrm{GHz}$, respectively. The measured bandwidth enhancement factor is 2.45 and 1.46 for the proposed TIA and the series peaking one, respectively. Fig. 6(b) illustrates the approximately linear phase response for both TIAs. Fig. 7 shows the transimpedance response for different power supplies. It is demonstrated that the proposed TIA can normally work $1.2 \mathrm{~V}$ and further below 1V. Fig. 8 shows the measured frequency response for the proposed TIA under $1.2 \mathrm{~V}$, where s11 and s22 are almost below $-10 \mathrm{~dB}$. When the supply voltage is $1.2 \mathrm{~V}$, the measured eye diagrams by PRBS of $2^{\wedge 11}-1$ are shown in Fig. 9. It can achieve the highest data rate of $12.5 \mathrm{~Gb} / \mathrm{s}$.

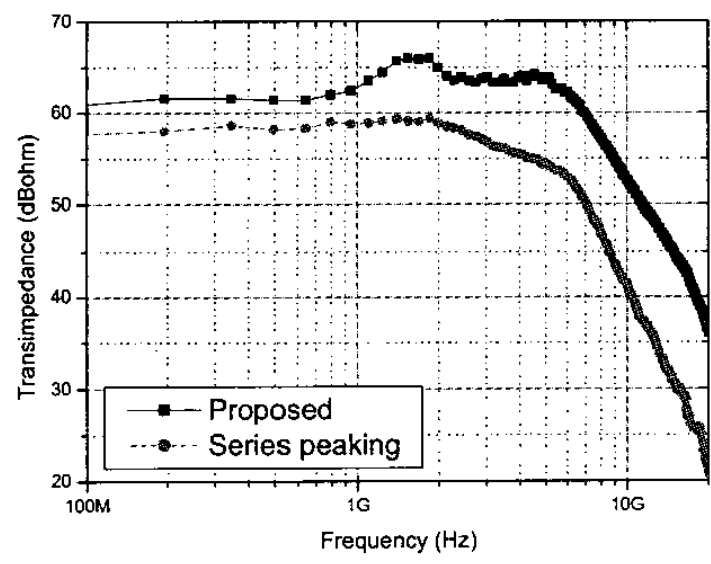

(a)

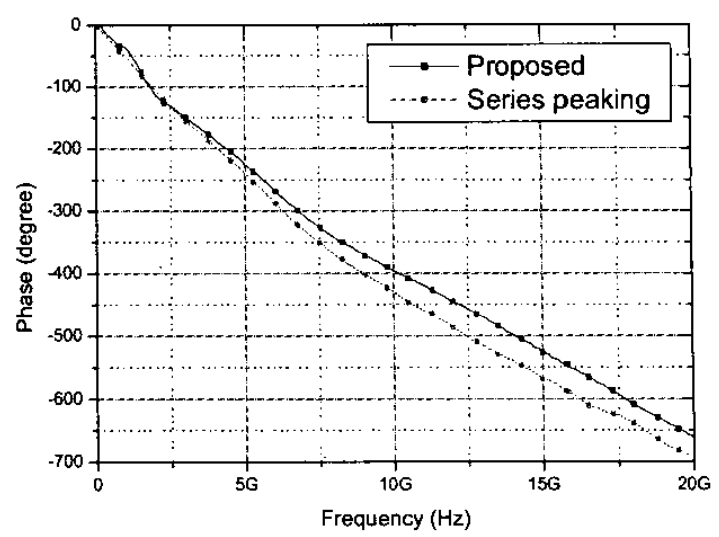

(b)

Fig. 6 (a) the measured transimpedance gain for the proposed TIA and the series peaking one.

(b) the measured phase response for the proposed TIA and the series peaking one.

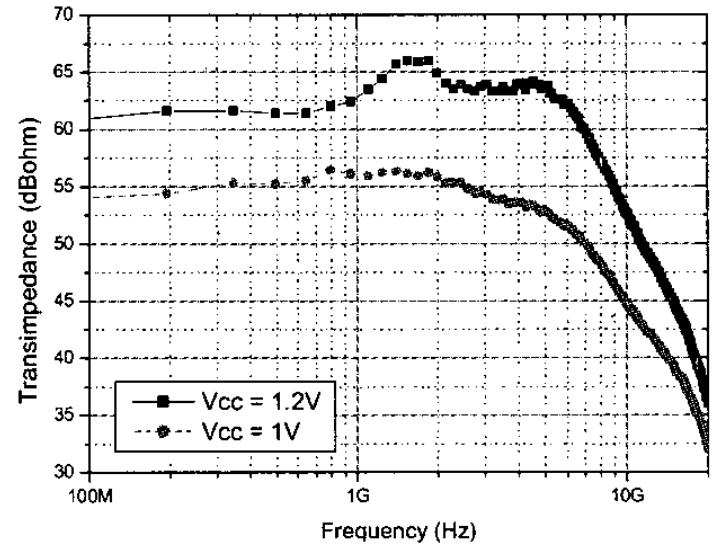

Fig. 7 Measured gain of the proposed TIA is $54.3 \mathrm{~dB} \Omega$ and $61.6 \mathrm{~dB} \Omega$ for $1 \mathrm{~V}$ and $1.2 \mathrm{~V}$ supply voltages, respectively.

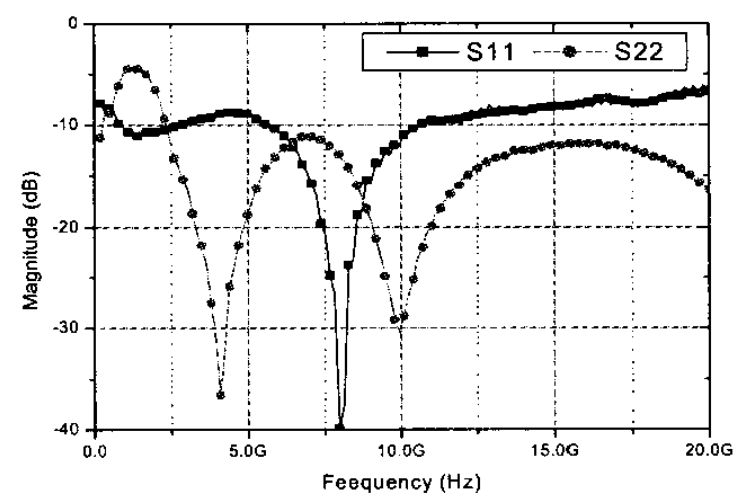

Fig. 8 Measured frequency response for the proposed TIA with the supply voltage $1.2 \mathrm{~V}$ 


\section{Conclusions}

Table I summarizes the measured performances of the proposed TIA, compared with other literatures. This TIA can operate under the supply voltage of $1.2 \mathrm{~V}$ and $18 \mathrm{~mW}$ power dissipations. To authors' knowledge, it is the first $10 \mathrm{~Gb} / \mathrm{s}$ SiGe transimpedance amplifier (TIA) with $1.2 \mathrm{~V}$ supply voltage. By using both the inductive series and shunting peaking techniques, the gain of $61.6 \mathrm{~dB} \Omega$ and the bandwidth of $7.4 \mathrm{GHz}$ can be achieved for $10 \mathrm{~GB} / \mathrm{s}$ applications.

\section{Acknowledgement}

The authors would like to thank National Chip Implementation Center for chip implementation. Special thanks to Mr. Wen, Mr. Chiu, and Dr. Huang of National Nano Device Laboratory team for measurement supporting. This work was supported in part by MediaTek Inc.

\section{References}

[1]. B. Razavi, "A $622 \mathrm{Mb} / \mathrm{s} \quad 4.5 \mathrm{pA} / \sqrt{\mathrm{Hz}} \quad \mathrm{CMOS}$ transimpedance amplifier," ISSCC Tech. Dig. pp. 162 $-163,453$, Feb. 2000.

[2]. W. Z. Chen and C. H. Lu, "A 2.5 Gbps CMOS optical receiver analog front-end," CICC, 2002. Proceedings of the IEEE 2002, pp. 359 -362, May 2002.

[3]. J. Lee, S-J. Song, S. M. Park, C-M. Nam, Y-S. Kwon, and $\mathrm{H}-\mathrm{J}$. Yoo, "A multichip on oxide of $1 \mathrm{~Gb} / \mathrm{s} 80 \mathrm{~dB}$ fully-differential CMOS transimpedance amplifier for optical interconnect applications," ISSCC Tech. Dig. pp. $80-447$, Feb. 2002.

[4]. H. H. Kim, S. Chandrasekhar, C. A. Burrus, and J, Bauman, "A Si BiCMOS transimpedance amplifier for 10-Gb/s SONET receiver," IEEE J. Solid-State Circuits, vol. 36, pp. $769-776$, May 2001.

[5]. B. Analui and H. Ali, "Multi-pole bandwidth enhancement technique for transimpedance amplifier," ESSCIRC 2002, Session C16, pp. 303-306, Sept. 2002

[6]. A. K. Petersen, et al.,"Front-end CMOS chipset for 10 Gb/s communication,"RFIC 2002, pp. 93-96, June 2002.

[7]. B. Razavi, Y. Ota, and R. G Swartz, "Design techniques for low-voltage high-speed digital bipolar circuits," IEEE J. Solid-State Circuits, vol. 29, pp. 332 -339, March 1994.

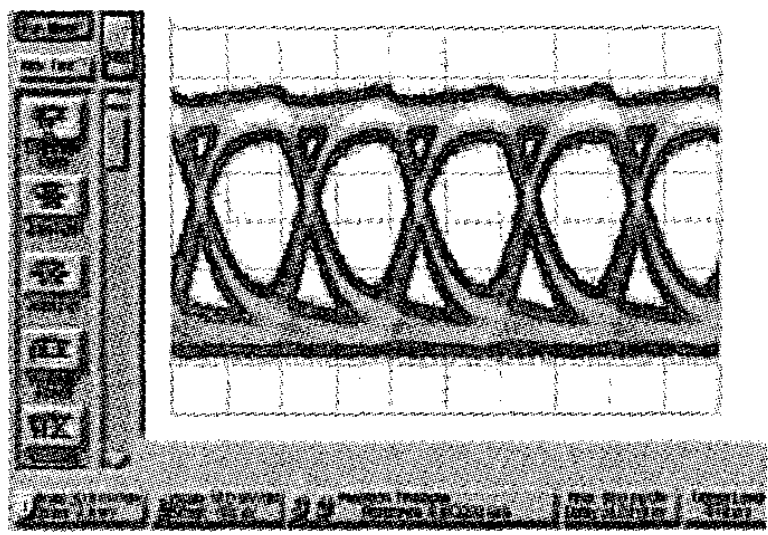

(a)

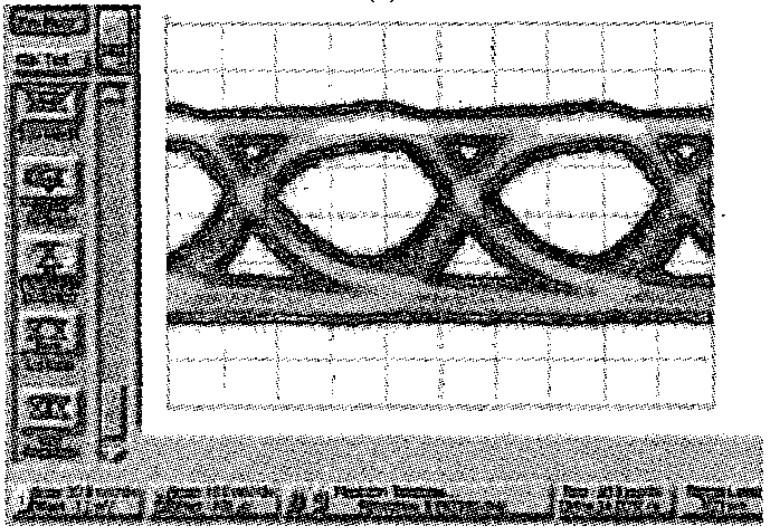

(b)

Fig. 9 Measured eye-diagrams for the proposed TIA with input equivalent current of $100 \mathrm{uA} \mathrm{A}_{\mathrm{p}-\mathrm{p}}, 1.2 \mathrm{~V}$ supply, and input data

(a) $10 \mathrm{~Gb} / \mathrm{s}$ of PRBS $2^{\wedge 1}-1$ (Horizontal scale: $50 \mathrm{ps} / \mathrm{div}$, vertical scale: $20 \mathrm{mV} / \mathrm{div}$ ).

(b) $12.5 \mathrm{~Gb} / \mathrm{s}$ of PRBS $2^{\wedge 31}-1$ (Horizontal scale: $20 \mathrm{ps} / \mathrm{div}$, vertical scale: $20 \mathrm{mV} / \mathrm{div}$ ).

[8]. S. S. Mohan, M. M. Hershenson, S. P. Boyd, and T. H. Lee, "Bandwidth extension in CMOS with optimized on-chip inductors," IEEE J. Solid-State Circuits, vol. 35, pp. $346-335$, March 2000

Table I. Performance summary (X: not mentioned)

\begin{tabular}{|l|c|c|c|c|c|c|}
\hline Reference & ISSCC00[1] & CICC02[2] & ISSCC02[3] & JSSC01[4] & ESSCIRC02 [5] & This work \\
\hline Technology & $\begin{array}{c}0.6 \mathrm{um} \\
\text { CMOS }\end{array}$ & $\begin{array}{c}0.35 \mathrm{um} \\
\text { CMOS }\end{array}$ & $\begin{array}{c}0.25 \mathrm{um} \\
\text { CMOS }\end{array}$ & $\begin{array}{c}0.25 \mathrm{um} \\
\text { BiCMOS }\end{array}$ & $\begin{array}{c}0.18 \mathrm{um} \\
\text { BiCMOS }\end{array}$ & $\begin{array}{c}\text { 0.35um } \\
\text { BiCMOS }\end{array}$ \\
\hline Supply voltage & $3 \mathrm{~V}$ & $3 \mathrm{~V}$ & $2.5 \mathrm{~V}$ & $5 \mathrm{~V}$ & $2.5 \mathrm{~V}$ & $1.2 \mathrm{~V}$ \\
\hline Speed & $622 \mathrm{Mb} / \mathrm{s}$ & $2.5 \mathrm{~Gb} / \mathrm{s}$ & $1 \mathrm{~Gb} / \mathrm{s}$ & $10 \mathrm{~Gb} / \mathrm{s}$ & $10 \mathrm{~Gb} / \mathrm{s}$ & $10 \mathrm{~Gb} / \mathrm{s}$ \\
\hline Transimpedance gain & $8.7 \mathrm{k} \Omega$ & $530 \Omega$ & $10 \mathrm{k} \Omega$ & $560 \Omega$ & $500 \Omega$ & $1.2 \mathrm{k} \Omega$ \\
\hline PD Capacitance & $\mathrm{X}$ & $0.5 \mathrm{pF}$ & $1 \mathrm{pF}$ & $0.15 \mathrm{pF}$ & $0.5 \mathrm{pF}$ & $0.15 \mathrm{pF}$ \\
\hline $\begin{array}{l}\text { Input referred noise } \\
\text { current density }(/ \sqrt{\mathrm{Hz}})\end{array}$ & $4.5 \mathrm{pA}$ & $800 \mathrm{nA}$ & $130 \mathrm{nA}$ & $X$ & $17 \mathrm{pA}$ & $22 \mathrm{pA}$ \\
\hline Power dissipation & $\begin{array}{c}30 \mathrm{~mW} \\
\text { (Excluding buffer) }\end{array}$ & $25 \mathrm{~mW}$ & $27 \mathrm{~mW}$ & $\begin{array}{c}140 \mathrm{~mW} \\
(\text { Buffer: } 80 \mathrm{~mW})\end{array}$ & $138 \mathrm{~mW}$ & $18 \mathrm{~mW}$ \\
\hline Chip area & $0.2 \mathrm{~mm}^{2}$ & $0.02 \mathrm{~mm}^{2}$ & $0.02 \mathrm{~mm}^{2}$ & $X$ & $0.64 \mathrm{~mm}^{2}$ & $0.45 \mathrm{~mm}^{2}$ \\
\hline
\end{tabular}

